\title{
Editorial
}

\section{Authorship and the Consideration of Alternatives}

\section{Bruce E. Herbert and Wendi Arant Kaspar}

Originally, this editorial was intended as a discussion of authorship norms across disciplines - a seemingly harmless topic relevant to academic libraries given libraries provide guidance and access to knowledge in all disciplines and can serve as the linchpin in multi-, inter- or transdisciplinary research. Our explorations, though, led us in a different direction, one that has implications for this journal.

Research and scholarship have experienced profound changes across all disciplines. One of the more transformational shifts has been the movement toward more interdisciplinary research and collaborations across subjects and sectors. This necessitates the awareness of and ability to navigate different disciplinary norms and expectations. The scholarly practices of academic communities often have unique values and norms, including the structure and system of rewards; the nature of collaborative, interdependent, and solitary research practices; and the models and norms of authorship and acknowledgment.

This is of particular importance in librarianship. Palmer and Cragin (2008) argue that "Understanding the nature of information practices and their relation to the production of scholarship is important for both theoretical and applied work in library and information science (LIS)." ${ }^{1}$ Understanding authorship norms, while not making assumptions or imposing judgment on disciplinary practice, is crucial in order to work more effectively with faculty or guide students.

Our interest in the meaning of authorship in different disciplines was piqued by a study that examined how prolific some authors are:

Authorship is the coin of scholarship-and some researchers are minting a lot. We searched Scopus for authors who had published more than 72 papers (the equivalent of one paper every 5 days) in any one calendar year between 2000 and 2016, a figure that many would consider implausibly prolific. We found more than 9,000 individuals, and made every effort to count only 'full papers' - articles, conference papers, substantive comments and reviews-not editorials, letters to the editor and the like. We hoped that this could be a useful exercise in understanding what scientific authorship mean. ${ }^{2}$

The authors of this study acknowledge that the question here is not around ethical practice but around disciplinary standards and how authorship is considered. 


\section{Authorship Norms Within and Across Disciplines}

When it comes to authorship and acknowledgement practices, authorship norms are often tacit and inconsistent among collaborators. This can lead to misunderstandings and strained relationships:

A few years ago, I shared a graduate student and a publication with a friend of mine in the department. When it came time to finalize the manuscript, we discussed the order that the three of us should appear on the author list. Believing I was being generous, I offered my friend last authorship, as I was taught that this is the position of distinction awarded to the leader of the project. My friend (nearly insulted by the suggestion) was much more interested in first authorship, believing the order of authors indicates their level of contribution to the work. ${ }^{3}$

Who is listed as an author and how the order of authorship represents the contributions to the work is of critical importance because authorship on publications is evidence of scholarship and the basis of academic reputation. We have always found that collaborations are more successful when the team explicitly understands authorship expectations. Being explicit about authorship can be particularly important as the practices of research and scholarship continue to evolve towards greater interdisciplinarity in collaborative research.

It can be surprising, then, that there is a wide variation of authorship norms even among related academic communities. For instance, a survey of health science researchers revealed a diverse understanding of how authorship is determined:

For some researchers, definitions of "doing the work" turned out to be very broad with one participant attributing authorship to "anyone who has contributed something to the development of the paper." [A1] This included: writing the paper, developing methodology, engaging in statistical analysis, data analysis, problem-solving or doing technical assays. In some cases, this extended to those who did as little as contribute an idea, provide a venue or supply a reagent used in the work. ${ }^{4}$

After several months of discussion and reading, we have reached this conclusion... there are no norms! There are many experts and scholars from different disciplines and professions weighing in on this issue, every day (literally). No one seems to have a definitive answer. While each discipline may have traditions and practices, each publication within the discipline seems to be fairly different and it is left up to the discretion of the authors and the editors to hold to a standard that may or may not be articulated. Good luck!

This impression is supported by another recent study by Patience et al. exploring the results of a survey of more than 5,500 science authors about what roles earn authorship. ${ }^{5}$ They asked questions about 25 different aspects of writing, analyzing, executing, designing and supervising (their terms). The results varied by disciplines, stage of career and region, indicating that norms may not really exist.

The journal Nature alone seems to have a piece on authorship with almost every issue. If that isn't a measure of the changing practices, we don't know what is. 
With the lack of clear disciplinary norms of authorship, numerous journals or editorial groups have developed authorship guidelines:

- American Bar Association

- American Chemical Society

- American Mathematical Society

- American Sociological Association

- Association for Computing Machinery

- Council of Biology Editors

- Institute of Electrical and Electronics Engineers

- International Committee of Medical Journal Editors

- Society for Neuroscience

A more disciplinary agnostic group, Committee on Publishing Ethics (COPE) ${ }^{6}$ also provides useful guidance on specific situations and questions about authorship vs acknowledgement as well as other writing, editing, and publishing practices. Another piece (in Nature), offers a model that favors contributorship over authorship for the sake of replication and triangulation. ${ }^{7}$

These guidelines address fairly consistent issues of authorship including which project participants to include as authors on a paper, the appropriate conventions guiding the inclusion of senior researchers or lab managers on a papers, the use of alphabetical order vs. ordering by effort or contribution, and the role of the corresponding author.

\section{Ethical Practice in Authorship}

Authorship practices can raise important ethical issues. We have heard of cases that cite egalitarian sentiments that all authors have contributed equally to justify authorship based upon alphabetical order. It is a nice idea with a couple of major caveats: the first being the standard tyranny of the alphabet (which anyone with a last name beginning with a letter in the last half of the alphabet can probably provide numerous examples of why this is an issue; and the second being the systems and conventions that have been set up to publish, discover and acknowledge published information perpetuate the first author preference with all others being relegated to "et al."

Marušić, et al. did a systematic review of authorship practices across disciplines. The results identified a number of specific instances that researchers believe would qualify for authorship:

Five surveys asked for a single contribution that would qualify for authorship: the most frequent choice for psychologists was choice of statistical method and data analysis (55\%) [11], manuscript drafting for nursing professionals (53\%) [13], design of the study for postdoctoral fellows from different disciplines (92\%) [29], providing statistical advice on an ongoing basis for researchers at a medical school (92\%) [31] and data interpretation or doing $20-50 \%$ of the work for business/nonbusiness faculty (90\%) [33]. In the latter study, more business than non-business faculty would grant authorship for only final preparation and submission of a manuscript ( $44 \%$ vs. $21 \%){ }^{8}$ 
These types of studies can help codify ethical practice. As an example, the American Psychological Association's Code of Ethics ${ }^{9}$ provides some guidance around appropriate authorship vs. other contributions:

Psychologists take responsibility and credit, including authorship credit, only for work they have actually performed or to which they have substantially contributed.... Mere possession of an institutional position, such as department chair, does not justify authorship credit. Minor contributions to the research or to the writing for publications are acknowledged appropriately, such as in footnotes or in an introductory statement... Except under exceptional circumstances, a student is listed as principal author on any multiple-authored article that is substantially based on the student's doctoral dissertation.

Following these ethical practices is especially important when there are power differentials in authorship negotiations, such as faculty-student coauthorship. Welfare and Sacket showed that ethical codes can help guide authorship norms between faculty and students:

The participant responses revealed a discrepancy between current and recommended authorship practices and a high likelihood of disagreement about appropriate recognition for student collaborators. Students and faculty members agreed that students do not always get the authorship recognition they should but they disagreed on the relative importance of many contributions to a research project. ${ }^{10}$

Many other professional societies have created similar codes of ethical practices.

\section{Evolving Publishing Practices to Improve Research Integrity}

Trust in the results of academic scholarship relies on the integrity of the research practices that produce that scholarship. Research integrity includes the use of honest and verifiable methods in proposing, performing, and evaluating research; communicating research results with adherence to rules, regulations, and guidelines; and following commonly accepted professional codes or norms. ${ }^{11}$

Concerns about authorship on publications have arisen. For instance, "Health science journals have had long-standing concerns that the names that appear at the top of an article do not reflect its true authorship." ${ }^{12}$ In an emerging issue, there are some indications that authorship may also be for sale in predatory open access journals. ${ }^{13}$

To combat these types of issues, the National Academy of Sciences recommends ${ }^{14}$ that:

Societies and journals should develop clear disciplinary authorship standards. Standards should be based on the principle that those who have made a significant intellectual contribution are authors. Significant intellectual contributions can be made in the design or conceptualization of a study, the conduct of research, the analysis or interpretation of data, or the drafting or revising of a manuscript for intellectual content. Those who engage in these activities should be designated as authors of the reported work, and all authors should approve the final manuscript. 
In addition to specifying all authors, standards should (1) provide for the identification of one or more authors who assume responsibility for the entire work, (2) require disclosure of all author roles and contributions, and (3) specify that gift or honorary authorship, coercive authorship, ghost authorship, and omitting authors who have met the articulated standards are always unacceptable.

We see more and more journals adopting these standards, specifically disclosing the roles and contributions of authors on publications. For instance, publications in Nature now include an author contribution statement, ${ }^{15}$ which is a statement of responsibility that specifies the contribution of every author. In a recent editorial, Nature explained the rationale for their new reproducibility checklist, which included the requirements for the author contribution statement. ${ }^{16}$

Consortia Advancing Standards in Research Administration Information (CASRAI) has also provided a taxonomy of contributor roles. ${ }^{17} \mathrm{McNutt}$, et al., advocate for this model ${ }^{18}$ and provide recommendations for journals:

- Set clear standards for authorship

- Provide expectations for corresponding authors

- Commit to the use of the contributor roles taxonomy

- Require authors to adopt ORCID IDs and other identifiers

We applaud the efforts to codify best practices for publication authorship and feel confident that these steps will positively affect research integrity.

\section{Possibilities for Publishing in Librarianship and in College \& Research Libraries}

As editors and authors, these are issues that we must contend with as best we can, professionally and ethically. There are two values that should inform any course of action or any practice adopted:

1. Transparency of the process is critical is order to model open access and to ensure procedural justice. Having clearly articulated standards available to authors and contributors so that each can understand and frame their involvement and impact and represent it accordingly.

2. Aligning the rewards with desired practice is another key factor. Individuals continue to do those practices that are positively reinforced. In the academy, tenure standards are a major determinant of the choice of publication topic, venue and collaboration: "The assessment of publications during promotion and tenure decisions is a big part of the problem. Although these processes often have some mechanism to recognize a researcher's team contributions, the culture remains largely unchanged from 50 years ago." 19

With regard to this second piece, any changes will have to occur at the institutional or local level. However, considering the issue of transparency and procedural justice, there is a possible move that $C \mathcal{E} R L$ can consider and one that we should, as a steward of research in the profession: the shift away from "authorship" to the model recommended by CASRAI, emphasizing the diverse roles of all contributors. This would offer transparency into the creation of new knowledge and make each contributors' role clear, for purposes of giving credit and for fostering future research. 


\section{Notes}

1. Carole L. Palmer and Melissa H. Cragin. "Scholarship and Disciplinary Practices," Annual Review of Information Science and Technology 42, no. 1 (2008): 163-212, doi:10.1002/aris.2008.1440420112.

2. John PA Ioannidis, Richard Klavans, and Kevin W. Boyack. "Thousands of Scientists Publish a Paper Every Five Days," Nature (2018): 167, doi:10.1038/d41586-018-06185-8.

3. Mark A Schneegurt, "Awarding the Distinction of Authorship on a Scientific Paper," Transactions of the Kansas Academy of Science 117, nos. 3\&4 (2014): 270-272, doi:10.1660/062.117.0313.

4. Jackie M. Street, Wendy A. Rogers, Mark Israel, and Annette J. Braunack-Mayer. "Credit Where Credit Is Due? Regulation, Research Integrity and the Attribution of Authorship in the Health Sciences," Social Science $\mathcal{E}$ Medicine 70, no. 9 (2010): 1458-65, doi:10.1016/j.socscimed.2010.01.013.

5. Gregory S. Patience, Federico Galli, Paul A. Patience, Daria C. Boffito, "Intellectual Contributions Meriting Authorship: Survey Results from the Top Cited Authors Across All Science Categories," bioRxiv preprint posted 17 May 2018; doi: https://doi.org/10.1101/323519.

6. Committee on Publication Ethics, "Promoting Integrity in Research and its Publication" (2018), available online at https://publicationethics.org/authorship [accessed 7 December 2018].

7. Marcus R. Munafò and George Davey Smith. "Robust Research Needs Many Lines of Evidence," Nature 553 (2018): 399, doi:10.1038/d41586-018-01023-3.

8. Marušić, Ana, Lana Bošnjak, and Ana Jerončić. "A Systematic Review of Research on the Meaning, Ethics and Practices of Authorship across Scholarly Disciplines." PLoS One 6, no. 9 (2011): e23477. doi:10.1371/journal. pone.0023477.

9. American Psychological Association. "Section 8: Research and Publication, Section 8.12B Publication Credit." Ethical Principles of Psychologists and Code of Conduct (2016), available online at https://www.apa.org/ethics/code/\#812b [accessed 7 December 2018].

10. Laura E. Welfare and Corrine R. Sackett, "Authorship in Student-Faculty Collaborative Research: Perceptions of Current and Best Practices," Journal of Academic Ethics 8, no. 3 (2010): 199-215, doi: 10.1007/s10805-0109119-7.

11. National Institutes of Health, "What is Research Integrity," (2018), available online at https:/grants.nih. gov/policy/research_integrity/what-is.htm [accessed 7 December 2018].

12. Jackie M. Street, et. al, "Credit Where Credit is Due?"

13. Holly Else, "Predatory Journals Offer 'Authorship for Sale."” Times Higher Education (September 21, 2017), available online (with subscription) at https://www.timeshighereducation.com/news/predatory-journals-offerauthorship-sale [accessed 10 December 2018].

14. National Academies of Sciences, Engineering, and Medicine, Fostering Integrity in Research (2017), Washington, DC: The National Academies Press, https://doi.org/10.17226/21896.

15. "Nature Research Journals' Authorship Policy," Nature (2018), available online at https://www.nature.com/ authors/policies/authorship.html [accessed 10 December 2018].

16. “Checklists Work to Improve Science." Nature 556, 273-274 (2018), doi:10.1038/d41586-018-04590-7.

17. "CRediT," Consortia Advancing Standards in Research Administration Information (2018), available online at https://casrai.org/credit/ [accessed 10 December 2018].

18. Marcia K. McNutt, Monica Bradford, Jeffrey M. Drazen, Brooks Hanson, Bob Howard, Kathleen Hall Jamieson, Véronique Kiermer et al., "Transparency in Authors' Contributions and Responsibilities to Promote Integrity in Scientific Publication," Proceedings of the National Academy of Sciences 115, no. 11 (2018): 2557-60, available online at http://www.pnas.org/content/115/11/2557\#T1 [accessed 10 December 2018]

19. Gretchen L. Kiser, "No More First Authors, No More Last Authors," Nature 561, no. 7724 (2018): 435, doi:10.1038/d41586-018-06779-2. 